\title{
BMJ Open Association between the Japan Coma Scale scores at the scene of injury and in-hospital outcomes in trauma patients: an analysis from the nationwide trauma database in Japan
}

\author{
Yohei Okada, ${ }^{\oplus 1,2,3}$ Takeyuki Kiguchi, ${ }^{2}$ Ryoji liduka, ${ }^{3}$ Wataru Ishii, ${ }^{3}$ Taku Iwami, ${ }^{2}$ \\ Kaoru Koike ${ }^{1}$
}

To cite: Okada Y, Kiguchi T, liduka $\mathrm{R}$, et al. Association between the Japan Coma Scale scores at the scene of injury and in-hospital outcomes in trauma patients: an analysis from the nationwide trauma database in Japan. BMJ Open 2019;9:e029706. doi:10.1136/ bmjopen-2019-029706

- Prepublication history and additional material for this paper are available online. To view these files, please visit the journal online (http://dx.doi org/10.1136/bmjopen-2019029706).

Received 08 February 2019

Revised 12 June 2019 Accepted 09 July 2019

Check for updates

(c) Author(s) (or their employer(s)) 2019. Re-use permitted under CC BY-NC. No commercial re-use. See rights and permissions. Published by BMJ.

${ }^{1}$ Department of Primary Care and Emergency Medicine, Kyoto University Graduate School of Medicine, Kyoto, Japan

${ }^{2}$ Department of Preventive

Services, Graduate School of

Public Health, Kyoto University,

Kyoto, Japan

${ }^{3}$ Emergency and Critical Care Medicine, Japanese Red Cross Society, Kyoto Daini Hospital, Kyoto, Japan

Correspondence to

Dr Yohei Okada;

yokada-kyf@umin.ac.jp

\section{ABSTRACT}

Objective Japan Coma Scale (JCS) is a grading system used to evaluate disturbed consciousness in prehospital care settings. We aimed to identify the association between the JCS levels at the scene with in-hospital mortality, as well as the discrimination ability for the outcomes.

Design A retrospective cohort study based on the nationwide trauma database in Japan.

Setting Multicentre cohort study using data from the Japan Trauma Data Bank, which is a nationwide, prospective, observational trauma registry derived from 235 hospitals.

Participants Adult trauma victims transferred directly from the scene of injury to the hospital from January 2004 to December 2017 were eligible for inclusion.

Primary and secondary outcomes Primary outcome was the association between the JCS levels at the scene with in-hospital mortality. We conducted a multivariate logistic regression analysis to calculate the adjusted ORs of JCS levels with $95 \%$ Cls for in-hospital mortality. We also calculated the c-statistics for in-hospital mortality. Results 164723 patients were included in the analysis. In a multivariate logistic regression analysis, the corresponding adjusted ORs of JCS levels 2 and 3 referred to level 1 for in-hospital mortality were $4.1(95 \% \mathrm{Cl} 3.8$ to 4.4$)$ and $26.0(95 \% \mathrm{Cl} 24.8$ to 27.2$)$. The c-statistics of the JCS level for in-hospital mortality was $0.845(95 \% \mathrm{Cl}$ 0.842 to 0.849 ).

Conclusions Data from large multicentre prospective registry revealed strong associations of the JCS level at the scene of injury with in-hospital mortality as well as the good discriminatory performance for this outcome.

\section{INTRODUCTION}

\section{Background}

Trauma is a leading cause of death and disability across all ages worldwide. ${ }^{1}$ The rapid provision of field triage and emergency care by paramedics and rapid transport of patients from the scene of injury to a hospital can save lives, reduce the incidence of short-term
Strengths and limitations of this study

- This is the first study to evaluate the association between the Japan Coma Scale (JCS) on the scene and in-hospital outcomes among trauma patients listed in a Japanese nationwide database.

- Our sensitivity analysis indicated the robustness of the association between JCS and in-hospital mortality, despite the risk of a selection bias due to missing data values.

- Although the JCS measurement is a fundamental skill required for government-certified paramedics, the accuracy and inter-rater reliability of this scale might be subject to measurement bias.

disability and dramatically improve patients long-term outcomes. ${ }^{23}$

An immediate assessment of the trauma victim's level of consciousness is necessary during field triage. ${ }^{3}$ Although the Glasgow Coma Scale (GCS) is the most widely used instrument for such assessments by paramedics in the field and other medical professionals in various settings, ${ }^{3}$ this scale has been criticised for its complexity, and therefore it may not be practical for rapid and reliable assessments in prehospital settings. ${ }^{4}$ Accordingly, a more rapid and simple assessment tool is needed.

The Japan Coma Scale (JCS), a grading system to evaluate disturbed consciousness, was first published in $1974^{5-7}$ and has since been certified as a standard field tool assessing the level of consciousness by the Japan Fire and Disaster Management Agency. The JCS is used to classify a patient's level of consciousness into one of three categories determined by reactive eye-opening: level 1 indicates spontaneous eye-opening, level 


\begin{tabular}{ll}
\hline Table 1 Description of the JCS \\
\hline JCS level & Description \\
\hline Level 1 digit & Eye-opening spontaneously \\
Level 2 digits & Eye-opening to verbal or pain stimuli \\
Level 3 digits & No eye-opening to any stimuli \\
\hline
\end{tabular}

JCS, Japan Coma Scale.

2 indicates eye-opening in response to a verbal or pain stimulus and level 3 indicates no eye-opening (table 1).

\section{Objectives}

Currently, the JCS is used by paramedics in prehospital trauma care settings nationwide, and an association of this standard scale of consciousness with the prognosis of patients with stroke has been reported. ${ }^{78}$ However, little is known about the diagnostic ability of JCS for the assessment of trauma victims at scenes of injury. We aimed to identify the association between the JCS levels at the scene and in-hospital mortality, as well as JCS's discrimination ability for the studied outcomes.

\section{METHODS}

We have reported the methodology of this study according to the Strengthening the Reporting of Observational Studies in Epidemiology statement. ${ }^{9}$

\section{Study design and setting}

The included data were obtained from the Japan Trauma Data Bank (JTDB) data set 2018, which contains information from 235 hospitals across Japan, from January 2004 to December 2017. The JTDB is a nationwide, multicentre, prospective, observational trauma registry established in 2003 by the Trauma Registry Committee of the Japanese Association for the Surgery of Trauma and the Committee for Clinical Care Evaluation of the Japanese Association for Acute Care Medicine, ${ }^{10}$ and managed by the Japan Trauma Care and Research (JTCR), which is a non-profit organisation for trauma research. This registry was developed to improve the quality of trauma care by collecting prehospital information, clinical information during hospital stays, trauma diagnoses according to the Abbreviated Injury Scale (AIS), Injury Severity Score (ISS) and mortality outcomes. ${ }^{11}$ The data are compiled by administrators based on in-hospital charts and prehospital records, which are routinely submitted by paramedics to the hospitals. Nearly all institutions participating in the JTDB are government-certified tertiary emergency and critical care centres. ${ }^{10-12}$ Details of JTDB have been described previously. ${ }^{10-12}$ The anonymised data of JTDB were available for the institutional members of JTCR for the research, and we obtained it from the JTCR.

The ethics committee of each institution and the Japanese Association for the Surgery of Trauma approved participation in the JTDB; they also approved retrospective analyses using anonymised data from the JTDB.

\section{Study participants}

Adult trauma victims who were transferred directly from the scene of injury to the hospital and registered in the JTDB 2018 data set from January 2004 to December 2017 were considered eligible for inclusion. From the full JTDB 2018 data set, we excluded patients younger than 16 years and those who developed cardiac arrest (predefined as a heart rate of 0 and systolic blood pressure (SBP) of 0 ) at the scene of injury. We also excluded patients for whom the main mechanism of injury was a burn or unknown and those who were not transferred directly from the scene by ambulance with paramedics (ex. transferred by the helicopter with physician, transferred from another hospital or transferred without paramedics). Moreover, we also excluded patients with the missing information of prehospital JCS and in-hospital mortality.

\section{Data collection, variables and potential bias}

We collected and described the following clinical information from the JTDB 2018 data set: age, sex, type of injury (blunt or penetrating), vital signs at the scene as measured by paramedics (JCS, SBP and respiratory rate (RR)), GCS on arrival at the hospital, in-hospital mortality, AIS of the head and ISS. Patients were stratified by age into the following groups: <40, 40-49, 50-59, $60-69,70-79$ and $\geq 80$ years. They were additionally categorised by vital signs as follows: JCS, level 1, 2 and 3 digits; SBP, <90, 90-139, 140-199 and $\geq 200 \mathrm{~mm} \mathrm{Hg}$; RR, $<10,10-29$ and $\geq 30$ (bpm), based on the Japan Fire and Disaster Management Agency protocol for determining priority at the scene. Missing covariates were categorised as 'Unknown'.

The AIS is an anatomically based, consensus-derived and globally accepted severity scoring system used to classify individual injuries by body region (eg, region 1: head, 2: face, 3: neck, 4: thorax) and subsequently by relative severity on a 6 -point scale $\left(1\right.$, minor to 6 , maximal) ${ }^{13} 14$ Generally, an AIS score $\geq 3$ is considered a serious or more severe injury. ${ }^{13}$ The ISS is another globally accepted trauma severity scale associated with mortality. ISS scores are calculated using the AIS and range from 1 to 75 ; here, a score of $\geq 16$ is generally considered to indicate major trauma or polytrauma. ${ }^{13}$

The primary study outcome was in-hospital mortality, and the secondary outcomes were coma (GCS $\leq 8)$ on arrival at the hospital, a severe head injury (Head AIS $\geq 3$ ) and severe multiple trauma (ISS $\geq 16$ ). Some patients who lacked exposure and primary outcome data were excluded from the main analysis, which might have introduced selection bias. Therefore, we performed a sensitivity analysis to address the missing JCS and in-hospital mortality data as described in the next paragraph.

When JCS was published in 1974, the state of 'completely awake and alert' was not included in the JCS. ${ }^{5-7}$ In a strict sense, the JCS level 1 originally indicated spontaneous eye-opening, but not completely awake and alert. However, in recent decades, completely awake and alert patients have been described conventionally as 'Level 0 ' 
and have been treated generally within level 1 . Thus, in our study the JCS ' 0 ' in the JTDB, and it was combined with JCS level 1 patients.

\section{Patient and public involvement}

As the study was conducted based on secondary data, patients and/or the public were not involved. Ethical consideration was described as in the Study Design and Setting section.

\section{Statistical methods}

We describe the patients' characteristics and the distribution of the GCS score on arrival at the hospital at each JCS level. To identify the associations between the prehospital JCS levels with the primary and secondary outcomes, we set the JCS level as the explanatory variable and each outcome as an objective variable. Subsequently, we conducted a logistic regression analysis to generate crude ORs of the JCS levels with 95\% CIs. For potential confounders, we designated the covariates of age, sex, type of injury, SBP and RR at the scene which were categorised as above, and conducted a multivariable logistic regression analysis to calculate the adjusted ORs of JCS levels with $95 \%$ CIs. To evaluate the discriminatory performance of the JCS, we calculated the JCS c-statistics for the primary and secondary outcomes.

Additionally, we performed a sensitivity analysis to demonstrate the robustness of the primary outcome results and compensate for the risk of selection bias due to the exclusion of the patients with missing JCS and mortality data. We assumed three scenarios of missing data: (1) missing at random; (2) missing because of high JCS level or similar and death or a minor injury and survival; and (3) the most conservative scenario against the assumed association. In assumption 1, the missing data were assumed to have been distributed as observed for eligible patients, and we used random imputation to compensate for the missing data using this distribution. In assumption 2, either all missing JCS data were imputed as level 1 and all missing outcomes were recorded as survival (assumption 2a), or all were imputed as level 3 and death, respectively (assumption 2b). In assumption 3 , all missing data were imputed as level 3 and survival (assumption 3a) or as level 1 and death (assumption $3 \mathrm{~b})$. We analysed the primary outcome under each assumption.

We did not calculate a precise sample size because our analysis was secondary usage of already available data. However, based on the rule of 10 events per variable, ${ }^{15}$ we needed to include 160-200 events at least to be able to adjust the covariates designated in our analysis. The prevalence of the events (in-hospital mortality) was estimated at $10 \%$ according to the previous studies. ${ }^{10}{ }^{11}$ From this, we expected 1600-2000 cases to be a large enough sample size to calculate the point estimates with CIs sufficiently narrow to answer the research question.

All statistical results were calculated as point estimates with $95 \%$ CIs. All statistical analyses were performed using JMP Pro V.14 software (SAS Institute, Cary, NC, USA).

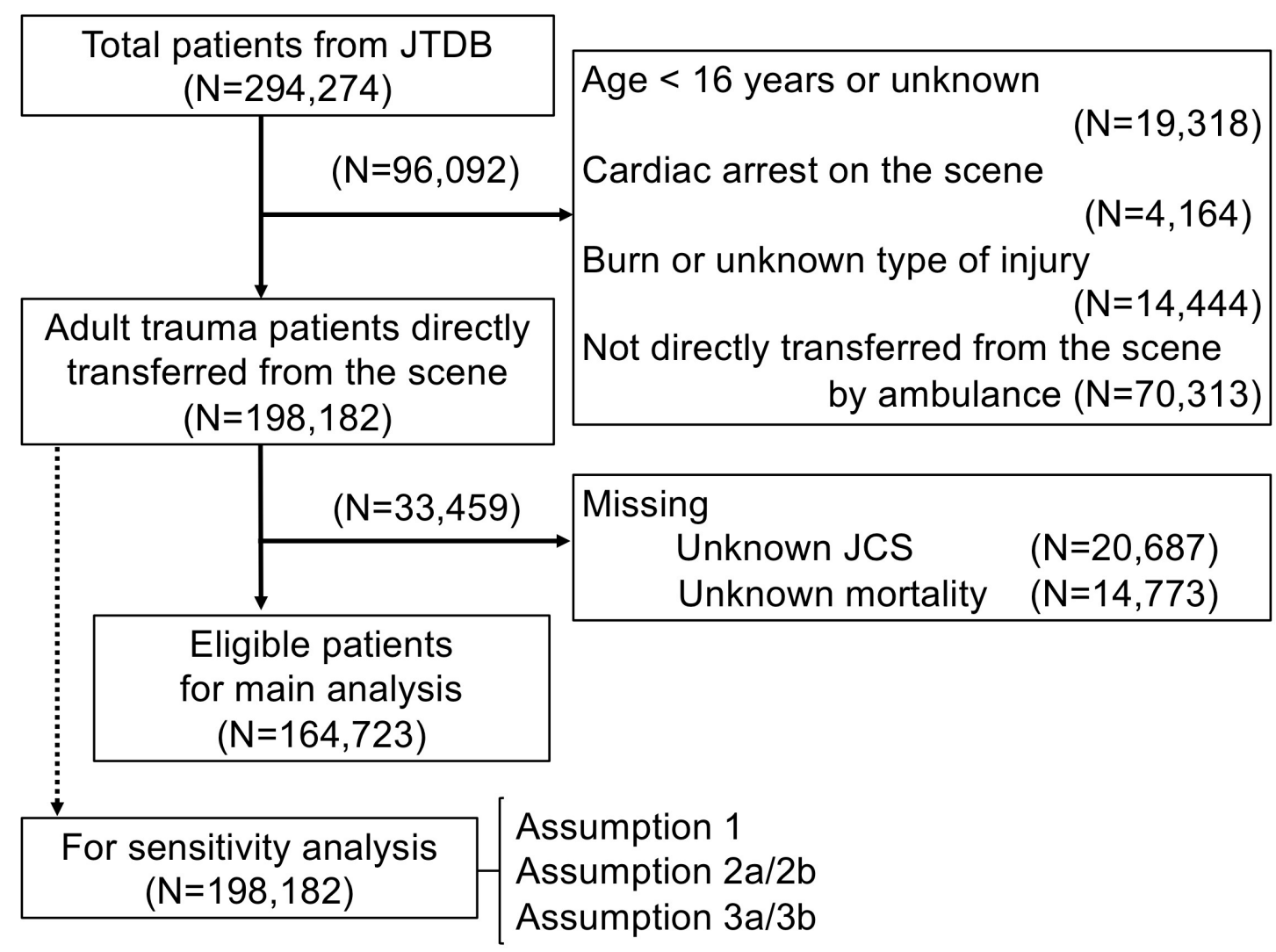

Figure 1 Flow chart of patient selection for the study. JCS, Japan Coma Scale; JTDB, Japan Trauma Data Bank. 
Table 2 Characteristics and prehospital information of the study participants

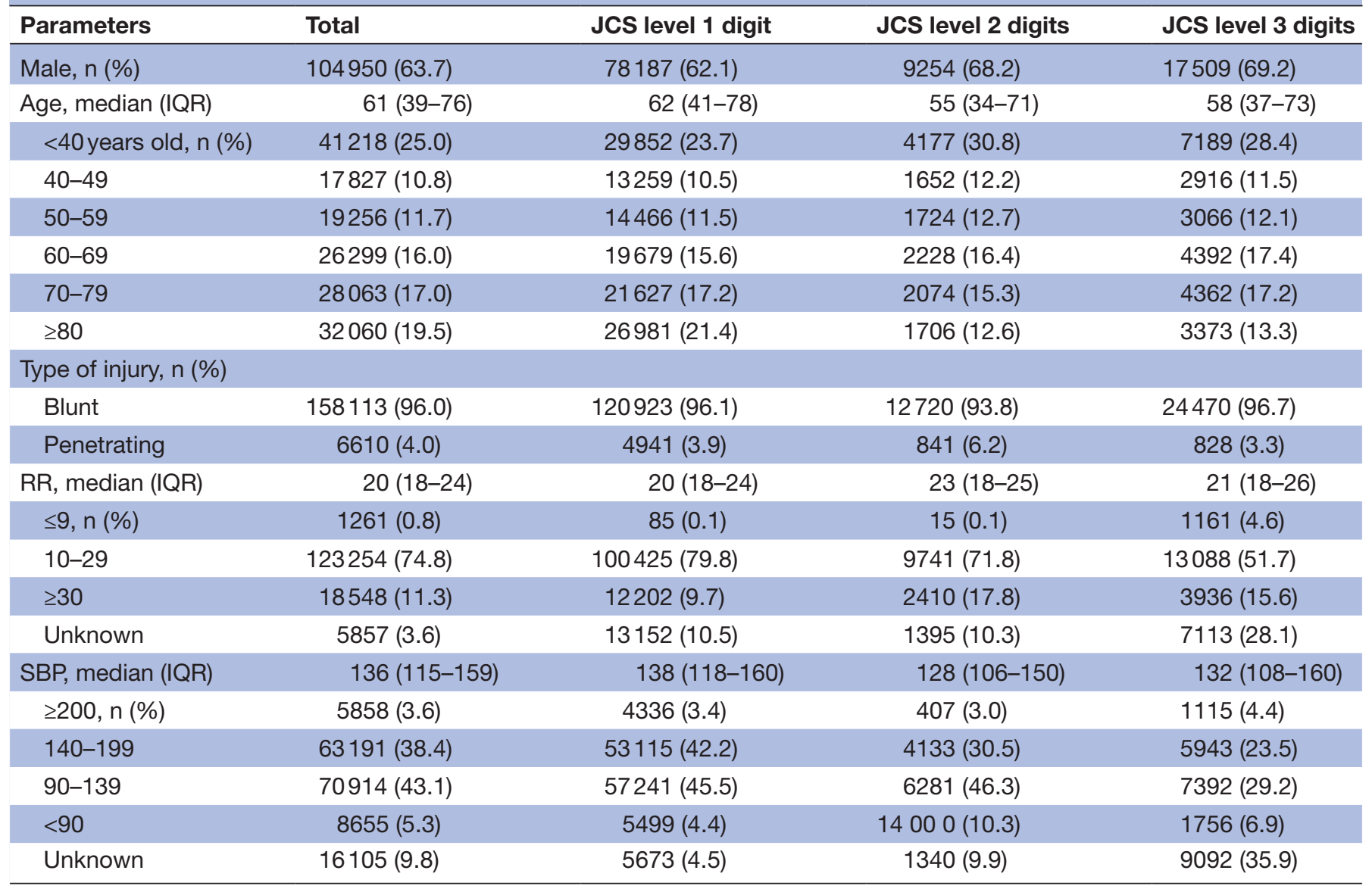

bpm, breaths per minute; JCS, Japan Coma Scale; RR, respiratory rate (bpm); SBP, systolic blood pressure $(\mathrm{mm} \mathrm{Hg})$.

\section{RESULTS}

\section{Patient characteristics}

Among the 294274 patients in the JTDB 2018, we excluded 19318 non-adult patients (<16 years or age unknown), 4164 patients who developed cardiac arrest at the scene of injury, 14444 patients with burn injuries or injuries of unknown mechanism and 70313 patients who were not directly transferred from the scene by ambulance. We also excluded 33459 patients with missing JCS $(n=20687)$ or mortality data $(n=14773)$. Finally, 164723 patients were included in the analysis (figure 1).

The patient's characteristics are described in table 2 . For the distribution between JCS at the scene and GCS on arrival at hospital, almost all JCS level 1 patients had a GCS of 14-15 on arrival at the hospital (83.4\%, 105 $017 / 25$ 298), while most JCS level 3 patients had a GCS $\leq 8(78.9 \%, 19112 / 25$ 298) (online supplementary file).

\section{Outcome}

The overall in-hospital mortality (primary outcome) was $10.9 \% \quad(n=17957)$. The distribution of primary and secondary outcomes by each JCS level is described (tables 2 and 3). Notably, a univariate logistic regression analysis of the associations of JCS levels with in-hospital mortality yielded crude ORs of 4.2 (95\% CI 3.9 to 4.5 ) and 36.7 (95\% CI 35.2 to 38.3) for levels 2 and 3, respectively, relative to level 1 (reference) (table 4 ). In a multivariate logistic regression analysis, the corresponding adjusted ORs were 4.1 (95\% CI 3.8 to 4.4) and 26.0 (95\% CI 24.8 to 27.2 ), respectively (table 4 ). Furthermore, the adjusted ORs of the JCS levels 2 and 3 compared with level 1 for the secondary outcomes as below: coma on arrival (GCS $\leq 8)$ (adjusted ORs: 11.6 (95\% CI 10.8 to 12.5 ) and 207.6 (95\% CI 195.5 to 220.4$)$ ), severe head injury (Head AIS 23) (adjusted ORs: 5.0 (95\% CI 4.8 to 5.2) and $13.4(95 \%$ CI 12.9 to 13.9$)$ ) and severe multiple trauma (ISS $\geq 16$ ) (adjusted ORs: 3.0 (95\% CI 2.9 to 3.1 ) and 8.9 (95\% CI 8.6 to 9.3$)$ ), respectively (tables 3 and 4 ). The adjusted ORs of the other parameters (injury type, age, sex, SBP, $\mathrm{RR}$ ) were described in another part (online supplementary file).

\section{Diagnostic ability}

The c-statistics of the JCS level for in-hospital mortality was 0.845 (95\% CI 0.842 to 0.849$)$. The c-statistics of the JCS level for secondary outcomes were as follows: coma on arrival (GCS $\leq 8)$ : 0.937 (95\% CI 0.935 to 0.939 ), severe head injury (Head AIS $\geq 3$ ): 0.685 (95\% CI 0.682 to 0.687 ) and severe multiple trauma (ISS $\geq 16$ ): 0.661 (95\% CI 0.659 to 0.663 ). Accordingly, the JCS appeared to exhibit good discriminatory performance for mortality and coma on arrival at the hospital. 
Table 3 In-hospital information and outcomes of the study participants

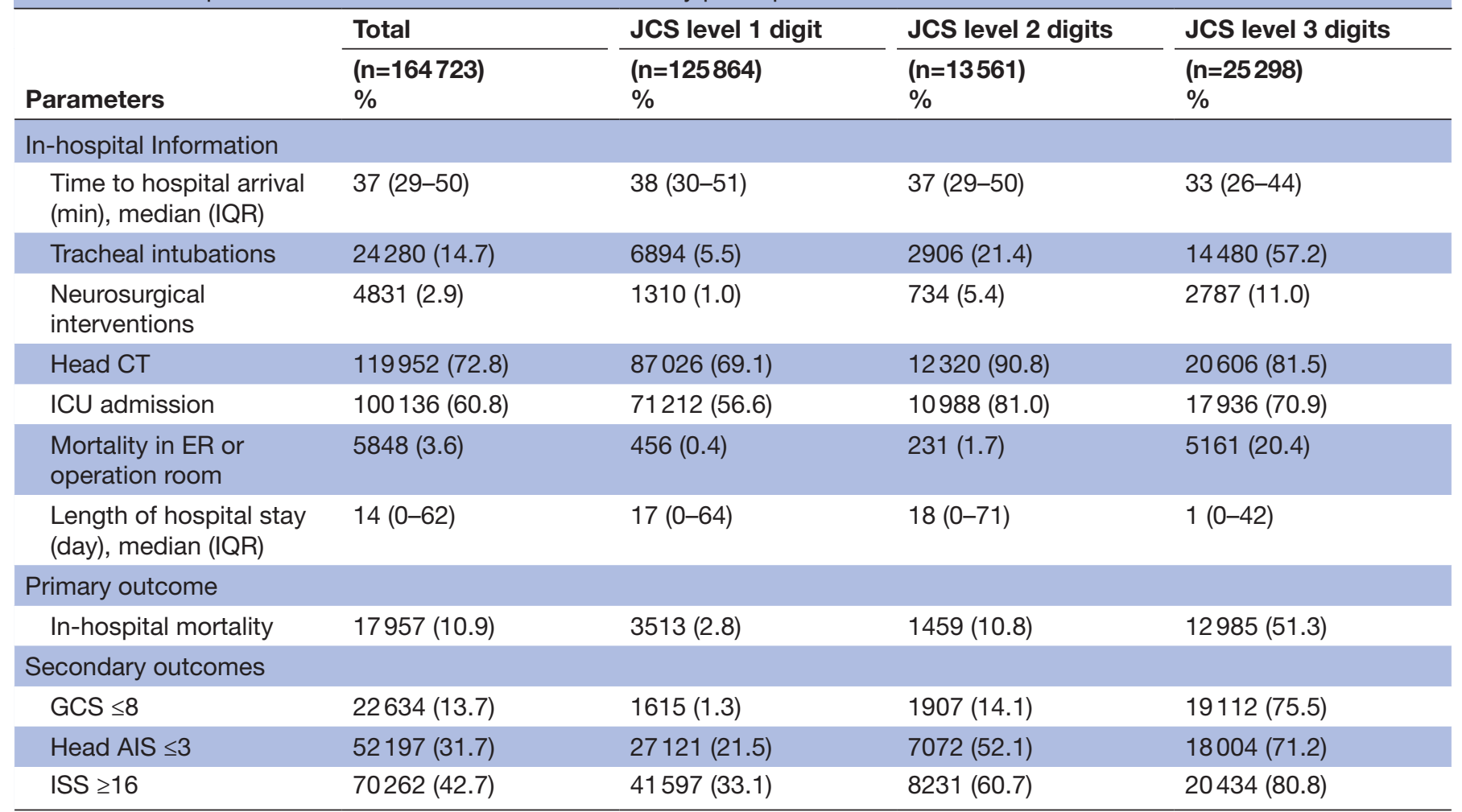

The number of missing GCS on arrival, Head AIS and ISS was 9910 (6.0\%), 3517 (2.1\%) and 3857 (2.3\%), respectively.

Time to hospital arrival denotes time from emergency call to hospital arrival.

AIS, Abbreviated Injury Scale; ER, emergency room; GCS, Glasgow Coma Scale; ICU, intensive care unit; ISS, Injury Severity Score; JCS, Japan Coma Scale.

\section{Sensitivity analysis}

In assumption 1, adjusted ORs of the JCS levels were as below: 2.7 (95\% CI 2.6 to 2.9 ) and 14.0 (95\% CI 13.5 to 14.5) for levels 2 and 3, respectively, relative to level 1. In all other assumptions, the JCS was also independently associated with in-hospital mortality (online supplementary file). These results demonstrate the robustness of this association, despite the missing values.

\section{DISCUSSION}

\section{Key observations}

To the best of our knowledge, this is the first study to evaluate the association between the prehospital JCS levels and in-hospital outcomes among trauma patients listed in a Japanese nationwide database. Notably, we demonstrated that the JCS level measured by paramedics on the scene correlated with in-hospital mortality, coma on arrival at hospital, severe head injury and severe multiple trauma. Furthermore, we showed that the prehospital JCS could clearly discriminate between coma on arrival at the hospital and in-hospital mortality.

\section{Interpretation and clinical implications}

Similar to our results, a previous report on the JCS and outcome among patients with stroke revealed a trend that as the JCS level increased, mortality increased, and functional outcomes worsened. ${ }^{78}$ According to the previous report and our results, it might be reasonably expected that the JCS on the scene could reflect the extent of neurological impairment and the severity of injury, as well as predict in-hospital mortality.

As noted above, the JCS was originally developed in Japan in 1974, concomitantly with the publication of the GCS. ${ }^{7}$ Although the JCS has since been used by paramedics throughout Japan as a standard scale of consciousness in prehospital trauma care, there is no literature to verify the clinical meanings of JCS among trauma patients. This study could be useful to paramedics using JCS by promoting the understanding of JCS and predicting the severity and mortality easily in the prehospital settings.

JCS is expected to have a different role from GCS in prehospital care. JCS consists of only one evaluation item (eye-opening in response to stimuli); thus, it has some strength with respect to its simplicity and rapidity. According to the protocol of Japanese standards for prehospital evaluation and care paramedics should triage the trauma patients at the scene of accident within the first $15 \mathrm{~s}$ to evaluate the JCS level while simultaneously conducting initial assessments of the airway, respiration and circulation. ${ }^{16}$ After a systemic physical assessment to identify life-threatening injuries, the paramedics determine the priority and destination hospital within 2 min 
Table 4 Analysis of the associations between the JCS levels with outcomes

\begin{tabular}{lll}
\hline Variables & Crude OR $(95 \% \mathrm{CI})$ & $\begin{array}{l}\text { Adjusted OR } \\
(\mathbf{9 5 \%} \mathbf{C l})\end{array}$ \\
\hline $\begin{array}{l}\text { Primary outcome } \\
\text { OR for in-hospital mortality }\end{array}$ & \\
JCS & & \\
Level 1 digit & (Reference) & (Reference) \\
Level 2 digits & $4.2(3.9$ to 4.5$)$ & $4.1(3.8$ to 4.4$)$ \\
Level 3 digits & $36.7(35.2$ to 38.3) & $26.0(24.8$ to \\
& & $27.2)$ \\
\hline
\end{tabular}

\section{Secondary outcomes}

OR for coma on arrival at hospital (GCS $\leq 8)$ JCS

$\begin{array}{lll}\text { Level } 1 \text { digit } & \text { (Reference) } & \text { (Reference) } \\ \text { Level } 2 \text { digits } & 12.3(11.5 \text { to 13.2) } & \begin{array}{l}11.6(10.8 \text { to } \\ 12.5)\end{array} \\ & & 207.6(195.5 \text { to } \\ \text { Level } 3 \text { digits } & 268.3(253.2 \text { to } & 220.4) \\ & 284.3) & \end{array}$

OR for severe head injury (Head AIS $\geq 3$ ) JCS

\begin{tabular}{|c|c|c|}
\hline Level 1 digit & (Reference) & (Reference) \\
\hline Level 2 digits & 4.0 (3.8 to 4.1$)$ & 5.0 (4.8 to 5.2$)$ \\
\hline Level 3 digits & 9.0 (8.7 to 9.3$)$ & $\begin{array}{l}13.4 \text { (12.9 to } \\
13.9)\end{array}$ \\
\hline \multicolumn{3}{|c|}{ OR for severe multiple trauma (ISS $\geq 16$ ) } \\
\hline Level 1 digit & (Reference) & (Reference) \\
\hline Level 2 digits & 3.2 (3.1 to 3.3$)$ & $3.0(2.9$ to 3.1$)$ \\
\hline Level 3 digits & 10.7 (10.3 to 11.1$)$ & 8.9 (8.6 to 9.3$)$ \\
\hline
\end{tabular}

Adjusted for age, sex, type of injury, and respiratory rate and systolic blood pressure.

AIS, Abbreviated Injury Scale; GCS, Glasgow Coma Scale; ISS, Injury Severity Score; JCS, Japan Coma Scale.

of arrival at the scene. If the patients were assessed as JCS level 3, the protocol recommend to transport the patients to the tertiary critical care centre. ${ }^{16}$ Therefore, in this setting, the severity of the patient's condition can be approximately and quickly evaluated and can be conveyed using only a single phrase, such as 'Level 3,' at the scene of the accident. ${ }^{7}$ Moreover, people who lack sufficient knowledge and experience may find interpreting the more complex GCS difficult, ${ }^{17-19}$ whereas the simpler JCS may be more comprehensible by non-medical staff members. Therefore, we thought that the JCS might be beneficial in simplicity and rapidity for a field triage.

Similar to the JCS, the simplified GCS has been suggested as an appropriate tool to assess the consciousness for field triage (eg, the motor component of the GCS (mGCS), simplified motor scale). ${ }^{3}$ The systematic review reported that these scales have good discrimination ability for in-hospital mortality (pooled area under the receiver operating characteristic curve (AUROC): 0.855
(95\% CI 0.822 to 0.888 ) in mGCS, 0.840 (95\% CI 0.802 to 0.878 ) in simplified motor scale, as well as total score of GCS (pooled AUROC: 0.877 (95\% CI 0.847 to 0.906$)$ ). ${ }^{3}$ Our results (JCS level for in-hospital mortality 0.845 (95\% CI 0.842 to 0.849$)$ ) were comparable to other scales, and it was expected to be useful in clinical settings as well as mGCS and simplified motor scale. Further research is needed to compare these scales.

While JCS has some advantages, it also has some limitations. As the other coma scale such as GCS might be incorrectly scored in some patients (eg, in the setting of intoxication) ${ }^{20}$ the JCS likely has the same caveats of measurement. In addition, although our study indicated that the JCS level 3 was associated with the severe head injury, it is not equivalent with a diagnosis of severe head injury. Unconsciousness can occur due to systemic insults, electrolyte abnormalities, intoxication, baseline psychiatric comorbidities and other causes.

Moreover, in our study, the c-statistics for the Head AIS $\geq 3$, and ISS $\geq 16$ were lower than that of in-hospital mortality; thus, JCS might not be a suitable instrument to discriminate the anatomical severity of the injury. One possible explanation was that Head AIS $\geq 3$, and ISS $\geq 16$ represent only anatomical severity in trauma. As these items do not include physiological aspects, many patients without disturbance of consciousness might have been included in Head AIS $\geq 3$, and ISS $\geq 16$. For example, although skull base fracture is coded as Head AIS: 3 it might not affect patients' consciousness or prognosis. Therefore, for the JCS to be clinically useful, we should understand the pathological process that may affect the eye-opening.

\section{Generalisability, strengths and limitations}

Because our data were derived from a nationwide trauma database, we believe that our findings can be generalised throughout Japan and possibly to other developed countries. Moreover, our sensitivity analysis indicated the robustness of the association between JCS and in-hospital mortality, despite the risk of a selection bias due to missing data values. These strengths notwithstanding, our study also had several limitations. First, few cases of penetrating injury were included, and thus the generalisability of our findings to penetrating injury cases remains unclear. Second, although the JCS measurement is a fundamental skill required for government-certified paramedics, the accuracy and inter-rater reliability of this scale might be subject to measurement bias. Third, we could not compare the clinical usefulness and accuracy of the JCS with other consciousness scales such as the simplified GCS. ${ }^{4}$ Fourth, mortality among trauma patients depends on many factors, and a wide range of pathologies. The JTDB did not include the detail of clinical course or the cause of mortality. These items should be considered to improve the understanding and how results are adopted in clinical settings. Further research is needed to evaluate the clinical usefulness and accuracy of the JCS relative to other consciousness scales. 


\section{CONCLUSIONS}

Data from large multicentre prospective registry study have revealed strong correlations of the JCS level at the scene of injury with in-hospital mortality and trauma severity. Furthermore, we demonstrated that the JCS had good discrimination abilities for both coma on arrival at hospital and in-hospital mortality.

Contributors YO contributed to the conception and design of this study, and writing the manuscript. TK, TI and KK gave some critical appraisal for discussion part of the draft. RI and WI contributed to data acquisition. KK, TI and RI supervised the whole work. All authors approved the manuscript for publication.

Funding The authors have not declared a specific grant for this research from any funding agency in the public, commercial or not-for-profit sectors.

Competing interests None declared.

Patient consent for publication Not required.

Ethics approval Ethics committees of the Japanese Association for the Surgery of Trauma approved the JTDB. The approval document is available on the JTDB website (https://www.jtcr-jatec.org/traumabank/dataroom/ethics2.htm). The ethics committee of the representative institution (National Defense Medical College Research Institute) approved the participation in the JTDB and retrospective analysis of deidentified data (ID No 2548).

Provenance and peer review Not commissioned; externally peer reviewed.

Data availability statement № data are available.

Open access This is an open access article distributed in accordance with the Creative Commons Attribution Non Commercial (CC BY-NC 4.0) license, which permits others to distribute, remix, adapt, build upon this work non-commercially, and license their derivative works on different terms, provided the original work is properly cited, appropriate credit is given, any changes made indicated, and the use is non-commercial. See: http://creativecommons.org/licenses/by-nc/4.0/.

\section{REFERENCES}

1. Trauma TAAftSo. Trauma facts. Available: http://www.aast.org/ trauma-facts [Accessed 3 Nov 2018].

2. Varghese M, Sasser S, Kellermann A. Prehospital trauma care systems: Geneva: World Health organization; 2005.

3. Chou R, Totten AM, Carney N, et al. Predictive utility of the total Glasgow coma scale versus the motor component of the Glasgow coma scale for identification of patients with serious traumatic injuries. Ann Emerg Med 2017;70:e6:143-57.

4. Thompson DO, Hurtado TR, Liao MM, et al. Validation of the simplified motor score in the out-of-hospital setting for the prediction of outcomes after traumatic brain injury. Ann Emerg Med 2011;58:417-25.

5. Ohta T, Kikuchi H, Hashi K, et al. Nizofenone administration in the acute stage following subarachnoid hemorrhage. Results of a multi-center controlled double-blind clinical study. J Neurosurg 1986;64:420-6.

6. Shigemori M, Abe T, Aruga T, et al. Guidelines for the management of severe head injury, 2nd edition guidelines from the guidelines Committee on the management of severe head injury, the Japan Society of Neurotraumatology. Neurol Med Chir 2012;52:1-30.

7. Shigematsu K, Nakano $\mathrm{H}$, Watanabe $\mathrm{Y}$. The eye response test alone is sufficient to predict stroke outcome--reintroduction of Japan Coma Scale: a cohort study. BMJ Open 2013;3:e002736.

8. Kurogi R, Kada A, Nishimura K, et al. Effect of treatment modality on in-hospital outcome in patients with subarachnoid hemorrhage: a nationwide study in Japan (J-ASPECT study). J Neurosurg 2018;128:1318-26.

9. von Elm E, Altman DG, Egger M, et al. The strengthening the reporting of observational studies in epidemiology (STROBE) statement: guidelines for reporting observational studies. Ann Intern Med 2007;147:573-7.

10. Hondo K, Shiraishi A, Fujie S, et al. In-Hospital trauma mortality has decreased in Japan possibly due to trauma education. J Am Coll Surg 2013;217:850-7.

11. Research JTCa. Japan trauma data bank report 2017, 2017. Available: https://www.jtcr-jatec.org/traumabank/dataroom/data/ JTDB2017e.pdf [Accessed 10 Oct 2018].

12. Endo A, Shiraishi A, Matsui $H$, et al. Assessment of progress in early trauma care in Japan over the past decade: achievements and areas for future improvement. J Am Coll Surg 2017;224:191-8.

13. Lecky F, Woodford M, Edwards A, et al. Trauma scoring systems and databases. Br J Anaesth 2014;113:286-94.

14. Tohira H, Jacobs I, Mountain D, et al. Comparisons of the outcome prediction performance of injury severity scoring tools using the abbreviated injury scale 90 update 98 (AIS 98) and 2005 update 2008 (AIS 2008). Ann Adv Automot Med 2011;55:255-65.

15. Peduzzi P, Concato J, Kemper E, et al. A simulation study of the number of events per variable in logistic regression analysis. $J$ Clin Epidemiol 1996;49:1373-9.

16. Mashiko K. Trauma systems in Japan: history, present status and future perspectives. J Nippon Med Sch 2005;72:194-202.

17. Winship C, Williams B, Boyle MJ. Should an alternative to the Glasgow coma scale be taught to paramedic students? Emerg Med J 2013;30:e19.

18. Santos WC, Vancini-Campanharo CR, Lopes MCBT, et al. Assessment of nurse's knowledge about Glasgow coma scale at a university hospital. Einstein 2016;14:213-8.

19. Basauhra Singh Harvinderjit Kaur a/p, Chong MC, Thambinayagam Hari Chandran a/l, et al. Assessing nurses knowledge of Glasgow coma scale in emergency and outpatient department. Nurs Res Pract 2016;2016:8056350:1-5.

20. Shahin H, Gopinath SP, Robertson CS. Influence of alcohol on early Glasgow coma scale in head-injured patients. J Trauma 2010;69:1176-81. 